\section{Rectourethral fistula}

\section{Iyad Khamaysi, Eisa Haj, Kamel Yassin}

Technion-Israel Institute of Technology, Haifa, Israel

A 73-year-old male had prostatic adenocarcinoma since 2003. After external-beam radiotherapy and cryotherapy had failed, the patient was treated with transrectal high-intensity focused ultrasound (HIFU). Because of recurrent hematuria, pneumaturia and urinary tract infection, a fistula to the urinary tract was suspected. Computed tomography raised the suspicion of rectourethral fistula. Sigmoidoscopy showed a large rectourethral fistula between the distal anterior rectal wall and the prostate bed. The urinary catheter and the inflated balloon could be easily inspected (Fig. 1A,B). No endoscopic attempt was made to close the fistula because of its size and location. The case was discussed in a multidisciplinary forum. The patient was sent for urinary and colonic diversion before transanal surgical fistula sealing.

Transrectal HIFU is a relatively new technology [1] capable of inducing instantaneous and irreversible coagulative necrosis in all biologic tissue via a thermal effect (absorption

Department of Gastroenterology and Hepatology, Rambam Health Care Campus, Rappaport Faculty of Medicine, Technion-Israel Institute of Technology, Haifa, Israel

Conflict of Interest: None

Correspondence to: Iyad Khamaysi, Director, Invasive Endoscopy Unit, Department of Gastroenterology and Hepatology, Rambam Health Care Campus, Rappaport Faculty of Medicine, Technion-Israel Institute of Technology, POB 9602, Haifa 31096, Israel,

e-mail:k_iyad@rambam.health.gov.il

Received 9 October 2018; accepted 4 November 2018; published online 7 December 2018

DOI: https://doi.org/10.20524/aog.2018.0335
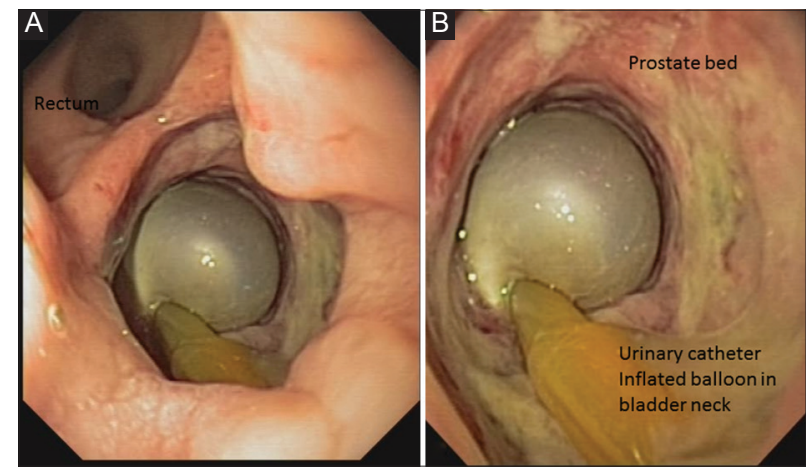

Figure 1 (A) Endoscopic view from distal rectum. The rectal lumen and the urinary catheter can be seen. (B) The scope is inside the prostate bed

of ultrasound energy converted into heat) and cavitation. Rectourethral fistula [2] is a possible but rare complication of the treatment of prostate cancer with transrectal HIFU treatment that inevitably requires stepped and complex reconstructive surgery.

\section{References}

1. Zhou YF. High intensity focused ultrasound in clinical tumor ablation. World J Clin Oncol 2011;2:8-27.

2. Choi JH, Jeon BG, Choi S-G, et al. Rectourethral fistula: systemic review of and experiences with various surgical treatment methods. Ann Coloproctol 2014;30:35-41. 